\title{
Comprehensive Project Management Framework using Machine Learning
}

\author{
Kalli Srinivasa Nageswara Prasad, M.V.Vijaya Saradhi
}

\begin{abstract}
Among the key reasons for the project management failures, unrealistic project schedules and ineffective utilization of the project conditions are key challenges. Irrespective of the size of the project and nature of project, there is imperative need for ensuring project conditions are effectively planned and there is holistic structure in place for managing the projects. There are many online solutions too which provides significant insights in to the system and its practices. The emergence of $A I$ and the machine learning models has created a paradigm shift in the way things are managed in the business and project functions. The decision-making related insights generated by the machine learning models can be a phenomenal impact for the organizations. It is evident from the depth of the study discussed in the report, that if effectively planned and right kind of machine learning models are chosen, there are prospective conditions for the models to be trained and an automated system be developed which can yield considerable results. Even for the implementation team, if there are too many ML models to be implemented as a process of detecting the right fit solutions and this study identifies the need for a more comprehensive and single-window system of machine learning based project management framework.
\end{abstract}

Key Words: ML based Project, AI based Project Management, AI based System Engineering, Sentimental Analysis based project models.

\section{INTRODUCTION}

Project management is one of the key practices that are helping the organizations to ensure that the projects executed by them are managed effectively. According to the studies that has focused on the project management practices and its implementation conditions, some of the key factors that are significant with the condition are the effective utilization of the resources, expected time duration for the completion of the task and the efforts that are integral to managing tasks and its subtasks[1]. managing the projects, there are also certain set of limitations that are encountered by the organizations. Firstly, the major and intrinsic reason for the failure of projects are because of the unrealistic project schedules. Irrespective of the size of the project and nature of project, there is imperative need for ensuring that the project conditions are effectively planned and there is holistic structure in place

Revised Version Manuscript Received on July 10, 2019.

Dr. Kalli Srinivasa Nageswara Prasad, Professor, Department CSE, Ramachandra College of Engineering, Eluru, Andhra Pradesh. Email: kallisnprasad@gmail.com

Dr.M.V.Vijaya Saradhi, Professor, Dept. Of CSE, ACE Engineering College, Ghatkesar, Hyderabad, India. Email: meduri.vsd@gmail.com
While there are significant benefits that are integral to

towards managing the projects.

The concept of project management practices, the tools and techniques too has evolved over period of time. Today, there are many cloud-based solutions that are available which supports the organizational teams in effective project management process [2]. There are certain contemporary solutions that compute the project schedule and provide estimated time lines based on the input provided in the tool. For instance, in the MS-Project tool, when the user provides the task duration and the resource allocated to the task and the cost for the resource, it provides the inputs in terms of duration start and end date, and the cost associated for the task, resource load factor etc. In Figure 1.

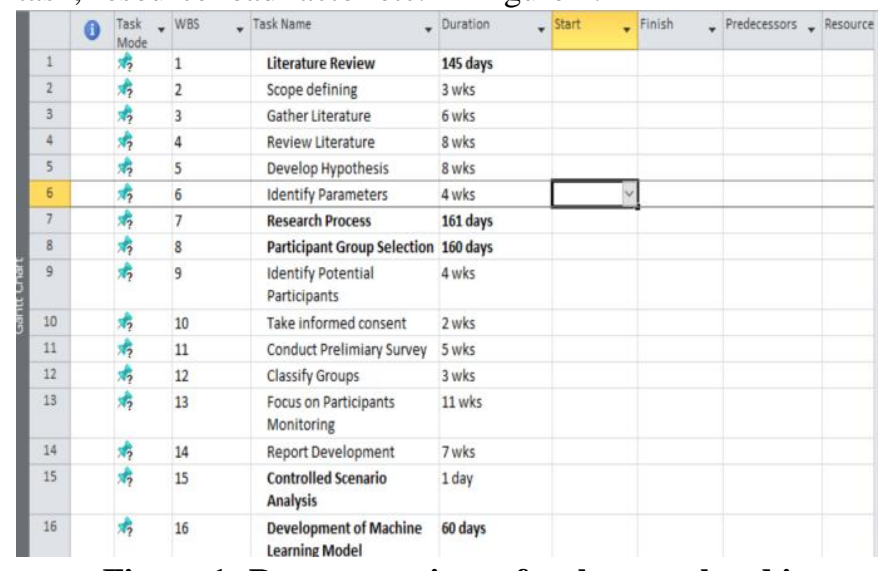

Figure 1: Representations of tasks completed in number of days

There are many online models too which provides significant insights in to the system and its practices. However, taking in to account the level of human intervention that is integral to the existing system of project management tools, there are potential chances that the human errors could be proving costly over the project conditions. Many of the online project management tools that are currently offered are either automated or the semi-automated systems [3].

In an illustrative scenario, when a project manager is working on a project schedule, there is a presumption from the resource based on his/her previous experience about the possible time frame for a task to be completed. However, there could be certain level of contingent conditions or the usual delay factors in the process that might consume more time for the project or actually the task might be completed within short time than the proposed time duration.

Majority of the current project management tools that are engaged by the organizations currently require certain level of estimation from the team

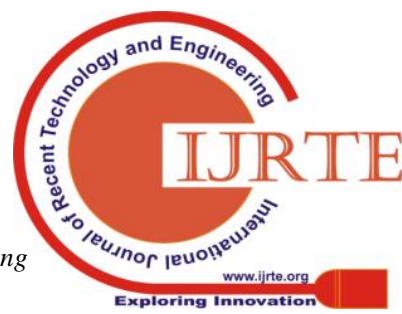




\section{Comprehensive Project Management Framework Using Machine Learning}

management. And the complete schedule is calculated based on the estimations fed by the human intervention.

With the increasing competition in the market, organizations are in need to manage their resources optimally and ensure the operational efficiency is high to complete the projects within the scheduled timelines. For the organizations handling multiple projects, the need for adhering to such timelines are pretty high. Taking such factors in to account, there is imperative need for more robust set of models that can improve the accuracy of the project schedules, guide the team with possible schedule impact related information [4].

The emergence of $\mathrm{AI}$ and the machine learning models has created a paradigm shift in the way things are managed in the business functions [5]. The decision-making related insights generated by the machine learning models can be a phenomenal impact for the organizations. This manuscript focuses on the significance of the machine learning models in the case of project management

In the further sections of this report, section-2 focuses on the literature review of the machine learning models used in the case of the project management. Followed by, in section-3 the discussions are carried out on how and what kind of solutions are available and the gaps that can be addressed by the future studies. Section-4 provides the conclusion of the project review.

\section{RELATED WORK}

Todays, project management scenarios are profoundly data-driven wherein the project managers need to augment the project insights. The AI based solutions can support the project managers in augmentation of the project conditions which can highlight the project risks, determination of the optimal allocation conditions and automation of project management tasks.

In the case of project execution and monitoring, one of the key tasks for the project managers are about updating the project status regularly. Usually in the case of such updates, the combination of text summarization/comments and the RAG (Red-Amber-Green) status is indicated. Red reflects on the failing project conditions, amber depicting the risk factors envisaged and the green indicating on-track of the project tasks. The role of machine learning solutions is considered significant in the case of automating the process of depicting the RAG status.

In the research study [6], the authors are view point that NLP (natural language processing) can be very effective in managing the classification automatically to the respective RAG status.

Authors of study [7] has focused upon the conditions wherein the project related information are evaluated in detail for the machine learning solutions implementation. The study has suggested the scope of implementing the instance-based learning models using the K-means algorithmic approach and the other regression model algorithms. Though the experimental inputs of the study do not provide in-depth study of the outcome, the scope and the conclusion discussed in the study highlights the possibility of using the machine learning solutions in the project management conditions.

However, the factor that has to be considered in such conditions are about understanding how the emerging techniques can be used for the distinct set of project management practices. For instance, focusing on whether the machine learning models can be more significant to one particular model or to all the kind of project management methods.

$\mathrm{AI}$ in the project management and how the contemporary AI practices in the project management can empower the project managers were discussed in [8]. The report highlights various critical aspects of the project management practices and how the artificial intelligence usage in the repetitive tasks of the project can support in more effective practice. The study while depicting the critical aspects of the project management like the task management, resource management and the cost management conditions, also discusses on how various stakeholder's integral to the project environment are to be taken in to account. By emphasizing on how the AI solutions can be potential model, the review paper provides insights on better ways in which the solutions can be pragmatic for routine implementation conditions.

It is evident from the depth of the study discussed in the report, that if effectively planned and right kind of machine learning models are chosen, there are prospective conditions for the models to be trained and an automated system be developed which can yield considerable results.

In an in-depth study carried out in [9], the authors of the study have carried out a literature review of the various AI based solutions for project management. Focusing on the distinct set of industry verticals and its relative developments, the authors examined the potential areas wherein the solutions can be significant. The key insights from the study are about focusing on the prediction rate accuracy for the project and towards identification of the critical success factors that are integral to the project environment.

Some of the models that are recommended in the study for the project success prediction are

- Adaptive Boosting Neural networks

- Evolutionary Fuzzy Neural Inference Model

- Support Vector Machine

- K-Means Clustering

- Bootstrap aggregating neural networks

- Bayesian Model

- Neural Networks

- Fast Messy Genetic Algorithm

Towards identifying the critical path related success conditions, which are very significant in the project management practice, the following set of algorithms are discussed

- Genetic Algorithms

- Fuzzy Cognitive Mapes

- Neural Networks

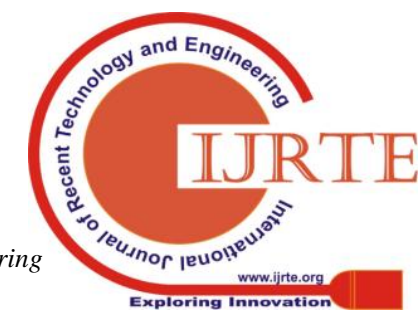


- Bayesian Model

The authors of the study have made subtle attempt in detailing what kind of algorithm models can be more effective in addressing the respective conditions of the project management and the best practices that can be focused upon. [9]

Focusing on a live project management scenario in a software project management environment, the study [10] has focused in depth on how the practical implementation of the machine learning model can address the issues. The methodical approach of conventional study wherein the scope of work is analyzed in a real-time condition, followed by focusing on the key aspects that support in evaluation process for the study provides critical insight on how the machine learning based models can be imperative to the project management practices. But one of the key factors that has to be taken in to account in the case of the project management conditions evaluated in the case scenario are about agile project management conditions. Wherein the proposed solutions or the framework should be tested to the practices of the other models of project management like the waterfall, Spiral model, Prototype modeling kind of project management practices.

Amazon web services (AWS) which offers robust cloud-based services for the distinct set of application solutions and project conditions, discussed about the viability of using the machine learning based solutions and effective project management. [11]

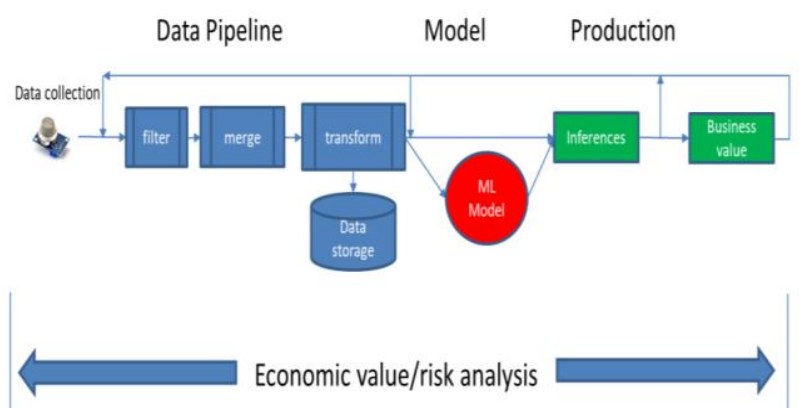

Figure 2: An Economic View of ML Projects

The Figure 2, indicates the economic dimension of using the machine learning based project conditions. If such integrated insights are developed from the project environment, it shall support in having more detailed insight in to vivid set of factors that plays a vital role in the system implementation and the key areas wherein the project needs more attention and the value addition. The spectrum of inputs discussed in the study if can be practically considered in the system of developing contemporary range of ML model for the project management, it can add value to the project momentum and efficiency.

Construction and infrastructure development companies rely highly on the project management practices to ensure adherence to the planned milestones and completion dates of the project. Also, in many of the construction related projects, the delays are outcome of non-pragmatic schedules combined with the external uncertainties that impact the project conditions. However, if the project management models are implemented with machine learning models for predicting the on-going conditions and its relative impact on project schedule, it can help in more accurate planning and course correction measures as required. In [12], the study has focused upon usage of the questionnaire-based data garnered about delays in the construction project of a company and used it as training dataset for a machine learning model. Using the WEKA software, the other project related delays for the same company are tested, which indicates a potential and accurate model for development.

Irrespective of the size of the projects and the industry, if the right kind of metrics are chosen, there are potential ways in which the machine learning based solutions can be resourceful for the projects.

The other project study [13] that has focused on the construction domain has proposed more significant solution in terms of implementing the ML based project prediction system. For any of the massive construction projects, budget controls play a vital role. Focusing on such critical factor, in the study the authors has discussed the model of EAC (estimation at completion), wherein the project's total cost movement and monitoring is given importance. Executives focus on high-level decisions in a project, but there could be tactical or operational issues that might impact the decisions. Hence, taking such factors in to control, the combined solution of machine learning based prediction system is proposed in the study. Using the coupled intelligence models GHS (global harmony search) and the BF (brutal force) models integrated to machine learning, a contemporary solution is discussed. The experimental study of the model carried out over the construction project in UAE, has provided the desired outcome from the system.

Genetic algorithm (GA) based simulation of construction time for the construction project is discussed in [14]. The authors of the study have focused on the distinct metrics that are important towards completion of the project well within the estimated schedule and accordingly the necessary practices are assessed, which will help the organization in developing more robust and contemporary set of solution. The outcome of the proposed GA based solution indicates the viability of the model and the ease with which the completion of the projects can be estimated. Considering the phenomenon of significant delays in the construction projects, if the stakeholders can rely on the kind of proposed GA based simulation applications, it will help in improving the accuracy of the estimations and towards achieving the desired outcome.

One of the critical factors that impacts the project management tracking and implementation is about usage of the right kind of project management tools. With dozens of solutions available for the project management, it is important that the right kind of solution is identified and implemented which can help in addressing the requirements more effectively. In [15], the authors of the study have applied machine learning based sentiment analysis model towards identification of the more suitable kind of 


\section{Comprehensive Project Management Framework Using Machine Learning}

project management tool to suit the specific kind of project management conditions. The results from the outcome of the study indicates that the model has been very resourceful and effective in terms of choosing the right kind of solutions and the system.

Software engineering as an approach is the combination of various functional and non-functional factors that are integral to the process of learning and management. It is far more important for the organizations to have more robust approach in the case of executing the software engineering.

The research study [16] has focused on the dynamics of using the deep learning models in the case of the software engineering process. The results from the study indicate that predominantly the model of deep learning can be effective in many of the software engineering phases. But the study also highlights that the practical application of such a system can be more complex in terms of handling the deep learning techniques integral to the system.

Project controls and monitoring is one of the critical factors that make significant difference to the system development. Though if there is high level of accuracy in a project system, if there is lack of substantial monitoring system, the project might have more risks and complexities. Focusing on the critical aspect of the project control conditions, in [17], The implementation of new libraries for learning evaluation in project control with open source software tools, opens a field of research related to increase technological integration with IT project management tools are highlighted. If such effective measures are taken up at every step of the project management, the ways in which it can lead to sustainable development can be much higher.

In summarization of the related work, it can be stated that if effectively planned and implemented, in the case of the project management practices, there are profound ways in which the machine learning models can be potential solution. It is evident from the studies that many of the existing research studies has focused on the role of using the AI and the machine learning models over the project management practices. With the evolving trends of the project management, there is need for more structured and intuitive kind of prediction models that can be significant to predicting the critical aspects of a project like the cost, resource utilization, critical path, budget, time duration and the scope of effective completion for the tasks. One of the constraints that is observed in the current set works reviewed are about the discussion of the application model for multi-sector project fitment conditions. In the further section of this manuscript, a detailed discussion on how the multi-kind project related insights can be resourceful are focused upon.

\section{DISCUSSIONS \& RESULTS}

Project management practices play a vital role in the assessment of the project course, the timelines for the WBS and the project related costs, utilization of resources, critical path etc. according to the reports of the management studies, among the key reasons for the project failure, the unrealistic project schedule, combined with ineffective monitoring practices are major challenges. In the other dimension, choosing the right kind of the project management methodology too is very essential. For instance, in the case of the construction projects, the PMBOK might be resourceful whereas in the case of the other segment of projects PRINCE2 could be a resourceful model and for few projects the lean project management model can be more appropriate [18] [5] [8].

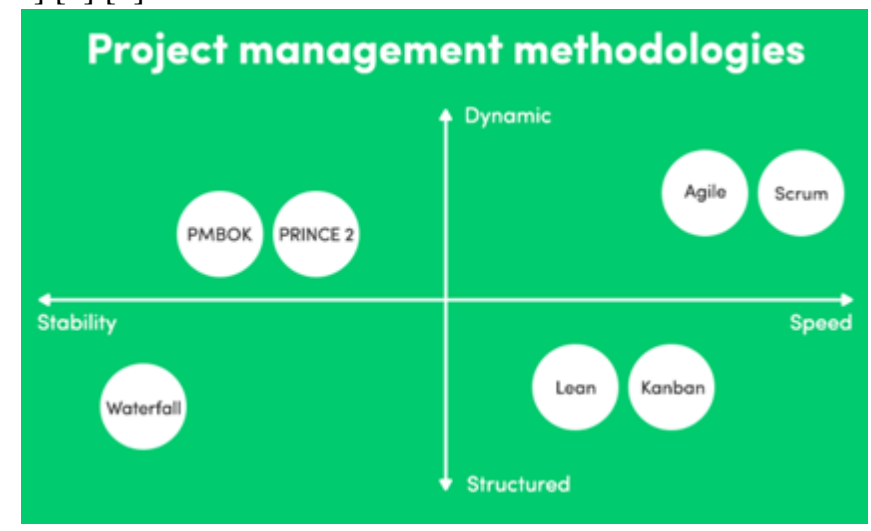

Figure 3: Project management methodologies

The Figure 3 mentioned above indicates the dynamics of various kind of project management methods. If the right kind of project management models are chosen, it will help in streamlining the solutions and towards developing a comprehensive system that can support in delivering the project successfully. Though in some of the earlier studies, the sentiment-analysis model has been proposed towards selection of a project management model, still if there is a comprehensive system which can support in selection of the model, it can be very effective.

Even for the implementation team, if there are too many ML models to be implemented as a process of detecting the right fit solutions, it shall be complex and there is need for more structured set of solution that can pave way to a comprehensive and integrated form.

For instance, in the case of a project management, irrespective of the size and the domain, certain common aspects and stages are

- Selection of a project management methodology

- Followed by, choosing the right kind of tool that can support in implementing the method

- Thirdly the process of estimating the project resources, schedule and time lines

- Monitoring and controlling the project scenario.

If there is an equated system that can focus on $t$ all the aforementioned stages and provide an integrated support, it can be more robust solution for the organizational process. But in many of the current proposals, the scope of a comprehensive system is lacking and it needs more emphatic system in place [19]

Predominantly, one of the key areas wherein the role of machine learning solutions should be significant is about tracking the progress of the project based on the daily

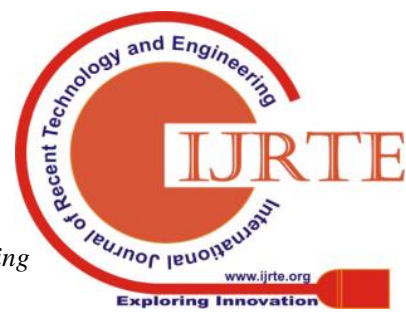


reports and the status updates. Using the combination of earlier data-based learning and the current possibilities, the system should be able to dispose options in terms of slack adjustment, or load management in the critical path and other such phenomenal conditions which might have more assertive set of actions on the project conditions. If there is such comprehensive model developed for the system, it might lead to more emphatic structure of project management.

When effectively used, machine learning models can deliver tangible business value in the project management domain. However, the objective and the scope of such implementation has to be more precise. There is huge gap in terms of the models that were proposed and the ones that are actually implemented. If the gap is reduced and there is more comprehensive system, the value addition from the machine learning model in the project management can be phenomenal.

By deploying a comprehensive system of machine learning model, it supports in transforming the potential of the ML in to realistic business value, as the process becomes the crux of the functional analysis rather than operating in silos. The comprehensive model can provide the decision makers with more clarity on the system, its functioning structure, the methods that are used and the overall outlook that can be achieved from the project. [20]

If the future works can focus on the system of dynamic machine learning solutions that can focus on all key fronts of system management like the resources, budget, timelines for the tasks, critical path, load management for resources, tracking and control, it can be a sustainable solution in the project management domain. With the quantum of machine learning models and the contemporary range of algorithms that are available in the ML 2.0, developing such a comprehensive system should be possible.

\section{CONCLUSION}

Leveraging on the resources, focusing on the emerging tools and techniques that can be resourceful for project management, combined with the realistic project schedules are some of the key areas wherein the system can be more prominent set of project management conditions for the project management using the machine learning models, it can lead to more sustainable solution.

In the present scenario, organizations need a more comprehensive solutions that can be a more of a single-window operation. Many of the existing models of machine learning based project management solutions were focusing on one or the set of project management factors, but a holistic solution is missing in the process. If there is a single interface solution developed, where in the project team feeds the probabilities, if the solution can develop a pattern for the project, such solutions might be more sustainable. For the future research studies, such solutions could be an option to explore. organizations. If there is a compact system developed for

\section{REFERENCES}

1. Cheng, Min-Yuan, Yu-Wei Wu, and Ching-Fang Wu. "Project success prediction using an evolutionary support vector machine inference model." Automation in Construction 19.3 (2010): 302-307.

2. Cheng, Min-Yuan, and Andreas FV Roy. "Evolutionary fuzzy decision model for construction management using support vector machine." Expert Systems with Applications 37.8 (2010) 6061-6069.

3. Cheng, Min-Yuan, et al. "A novel time-depended evolutionary fuzzy SVM inference model for estimating construction project at completion." Engineering Applications of Artificial Intelligence 25.4 (2012): 744-752.

4. Cheng, Min-Yuan, et al. "Estimate at completion for construction projects using evolutionary support vector machine inference model." Automation in Construction 19.5 (2010): 619-629.

5. Cheng, Min-Yuan, and Nhat-Duc Hoang. "Risk score inference for bridge maintenance project using evolutionary fuzzy least squares support vector machine." Journal of Computing in Civil $\begin{array}{lll}\text { Engineering } & 28.3 & \text { (2012): }\end{array}$ https://towardsdatascience.com/automating-project-management-w ith-deep-learning-bd5b851235eb

6. Miguel Pedroso, Application of Machine Learning Techniques in Project Management Tools. Information Systems and Computer Engineering, 2017, PP.1-11.

7. Amith Johnsonbabu. Reinventing the role of Project manager in the Artificial intelligence era. Project Management National Conference India. Sep.2017, PP. 1-11.

8. Martínez, D. Magaña, and Juan Carlos Fernández-Rodríguez. "Artificial intelligence applied to project success: a literature review." IJIMAI 3.5 (2015): 77-84.

9. Saglam, Sefa Oguz. "Applied machine learning: project management performance prediction at information technology company project management office." (2017).

10. V.M. Megler, Managing Machine Learning Projects, Amazon Web Services, Feb.2019, pp.1-35.

11. Asadi, Ahmad, Mohammed Alsubaey, and Charalampos Makatsoris. "A machine learning approach for predicting delays in construction logistics." International Journal of Advanced Logistics 4.2 (2015): 115-130.

12. Hares, Al, Enas Fathi Taher, and Cenk Budayan. "Estimation at Completion Simulation Using the Potential of Soft Computing Models: Case Study of Construction Engineering Projects." Symmetry 11.2 (2019): 190.

13. Kamoona, Kareem, Karrar Raoof, and Cenk Budayan. "Implementation of Genetic Algorithm Integrated with the Deep Neural Network for Estimating at Completion Simulation." Advances in Civil Engineering 2019 (2019).

14. Baro, R. A., et al. "Classification of project management tool reviews using machine learning-based sentiment analysis." IOP Conference Series: Materials Science and Engineering. Vol. 482 No. 1. IOP Publishing, 2019.

15. Xiaochen Li, He Jiang1,Zhilei Ren, Ge Li et.al., Deep Learning in Software Engineering. pp. 1-10.

16. García, José Alejandro Lugo, et al. "Project Control and Computational Intelligence: Trends and Challenges." International Journal of Computational Intelligence Systems 10.1 (2017) 320-335.

17. Weber, Philippe, et al. "Overview on Bayesian networks applications for dependability, risk analysis and maintenance areas." Engineering Applications of Artificial Intelligence 25.4 (2012): 671-682.

18. Wen, Jianfeng, et al. "Systematic literature review of machine learning based software development effort estimation models." Information and Software Technology 54.1 (2012): 41-59.

19. Malhotra, Yogesh. "AI, Machine Learning \& Deep Learning Risk Management \& Controls: Beyond Deep Learning and Generative Adversarial Networks: Model Risk Management in AI, Machine Learning \& Deep Learning: Princeton Presentations in AI-ML Risk Management \& Control Systems (Presentation Slides)." Machine Learning \& Deep Learning: Princeton Presentations in AI-ML Risk Management \& Control Systems (Presentation Slides) (April 21, 2018). Princeton Presentations in AI \& Machine Learning Risk Management \& Control Systems, 2018 Princeton Fintech \& Quant Conference, Princeton University. 2018. 